\title{
Generation of HLA-deficient platelets from hematopoietic progenitor cells
}

\author{
Constança Figueiredo, Lilia Goudeva, Peter A. Horn, Britta Eiz-Vesper, Rainer Blasczyk,* and \\ Axel Seltsam*
}

BACKGROUND: Exposure to allogeneic blood products often leads to the development of human leukocyte antigen (HLA) antibodies. Refractoriness to platelet (PLT) transfusion caused by alloimmunization against HLA Class I antigens constitutes a significant clinical problem.

STUDY DESIGN AND METHODS: We developed an RNA interference (RNAi)-based approach to silence the expression of HLA Class I molecules on PLTs derived from CD34+ progenitor cells. A lentiviral-based system was used to express short-hairpin RNA (shRNA) targeting $\beta 2$-microglobulin ( $\beta 2 \mathrm{~m}$ ) transcripts in CD34+ progenitor cells. Differentiation to PLTs was performed by incubating progenitor cells in the presence of thrombopoietin and interleukin-3.

RESULTS: The transduction of RNAi cassettes containing the sequences for shRNAs targeting $\beta 2 \mathrm{~m}$ caused up to $85 \%$ reduction of progenitor cells HLA Class I antigen expression, which was maintained in the culture-derived PLTs. The HLA-deficient PLTs derived from HLA-silenced CD34+ cells proved to be fully functional in in vitro tests when compared to peripheral blood-derived PLTs.

CONCLUSIONS: Our data show that in vitro generating HLA Class I-deficient PLTs from hematopoietic progenitor cells prove to be feasible. As malignancy risks associated with insertional mutagenesis are not to be expected in anucleated PLTs, provision of HLA-deficient PLTs from large-scale production units may become reality in the management of patients suffering from PLT transfusion refractoriness.
$\mathrm{P}$ latelet (PLT) transfusions are widely used for prevention and treatment of PLT dysfunction syndromes and thrombocytopenic bleeding in patients with hematologic diseases, solid tumors, or major surgical bleeding. ${ }^{1-4}$ Today, more than 1.5 million PLT products are transfused in the United States and 2.9 million in Europe each year. Lack of adequate posttransfusion PLT count increments-PLT refractoriness-is a major complication of long-term PLT supportive care experienced by $5 \%$ to $10 \%$ of chronic PLT recipients. ${ }^{5}$ PLT refractoriness may lead to fatal bleeding complications in thrombocytopenic patients. In most cases, the causes of refractoriness are clinical and pharmacologic. In those cases in which refractoriness is due to immune factors, human leukocyte antigen (HLA) antibodies are most frequently implicated. ${ }^{6}$ Frequent exposure to allogeneic blood products often leads to the development of anti-HLA by PLT recipients. It was shown

ABBREVIATIONS: $\beta 2 \mathrm{~m}=\beta 2$-microglobulin; eGFP $=$ enhanced green fluorescent protein; Flt3-L = Fms-like tyrosine kinase 3-ligand; FSC = forward scatter; GP(s) = glycoprotein(s); RNAi $=$ RNA interference; $\operatorname{sh} \beta 2 \mathrm{~m}=$ shRNAs specific for $\beta 2 \mathrm{~m}$; shNS = nonsense short-hairpin RNA; shRNA(s) = short-hairpin RNA(s); SSC $=$ side scatter; TPO = thrombopoietin.

From the Institute for Transfusion Medicine, Hannover Medical School, Hannover; the Institute for Transfusion Medicine, University Hospital Essen, Essen; and the German Red Cross Blood Services NSTOB, Institute Springe, Springe, Germany.

Address reprint requests to: Prof. Dr. Rainer Blasczyk, Institute for Transfusion Medicine, Carl-Neuberg Strasse1, 30625 Hannover, Germany; e-mail: blasczyk.rainer@mh-hannover.de.

*These authors contributed equally to the work.

CF designed and performed the experiments and wrote the manuscript, LG and BEV aided in performing experiments and analyzed and interpreted data, PAH analyzed and interpreted data, RB designed parts of the study and contributed to paper writing, and AS designed the study and wrote the manuscript.

Received for publication August 17, 2009; revision received

February 1, 2010, and accepted February 3, 2010.

doi: 10.1111/j.1537-2995.2010.02644.x

TRANSFUSION 2010;50:1690-1701. 
that those antibodies target few epitopes of HLA Class I molecules. ${ }^{7}$ Two validated strategies to select effective PLTs for alloimmunized refractory patients are the selection of HLA-matched PLT donors from HLA-typed donor registries and the use of manual or automated PLT crossmatching. Both strategies, which require significant organizational and financial resources, ${ }^{5}$ can provide successful PLT support in approximately two-thirds of transfusions. However, they may not work in cases with highly alloimmunized patients presenting with HLA Class I antibodies of broad specificity. In these situations, it would be desirable to have PLT units in which the antigenicity of HLA Class I has been eliminated from the PLT surface.

Hematopoietic stem cells and progenitor cells have emerged as an essential cell type in regenerative medicine. PLTs have been successfully generated from human cord blood and marrow CD34+ cells. ${ }^{8,9} \mathrm{CD} 34+$ cell-derived PLTs exhibited features quite similar to peripheral bloodderived PLTs in terms of morphology and function. The culture systems used to expand and differentiate hematopoietic progenitor cells (HPCs) appear to be applicable for large-scale generation of PLTs for future clinical use. In addition, recent reports about in vitro production of functional PLTs by differentiated mouse embryonic stem cells suggest the potential utility of embryonic stem cellderived PLTs as a substitute for PLT transfusion. ${ }^{10-12} \mathrm{Com}$ bined with the genetic manipulability of stem cells and HPCs, these approaches offer the opportunity to generate genetically engineered PLTs for clinical transfusion.

RNA interference (RNAi) has become a powerful tool in molecular medicine. RNAi is an evolutionarily conserved mechanism for silencing gene expression by targeted degradation of mRNA. Short double-stranded RNAs, known as small interfering RNAs, are incorporated into an RNA-induced silencing complex that promotes the degradation of RNA containing a homologous sequence. Lentiviral-based vectors have proved to be effective vehicles for the delivery of RNAi cassettes into mammalian cells, which allow a stable silencing of the target gene expression. ${ }^{13-15}$ Previously, we have shown the feasibility to regulate cell surface expression of HLA Class I molecules using a RNAi-based method. ${ }^{16}$ In this study, we used an RNAi-based approach to permanently silence the expression of HLA Class I molecules on PLTs derived from CD34+ progenitor cells and demonstrated in vitro that these HLA Class I-deficient PLTs are functionally equivalent to blood-derived PLTs.

\section{MATERIALS AND METHODS}

\section{CD34+ cell isolation and enrichment}

Peripheral blood CD34+ progenitor cells were isolated from leukapheresis samples of eight healthy individuals selected as donors for allogeneic stem cell transplanta- tion. Informed consent as approved by the local ethics committee of Hannover Medical School was obtained from all donors. The donors received $10 \mu \mathrm{g} / \mathrm{kg}$ body weight recombinant granulocyte-colony-stimulating factor (G-CSF; Lenogastrim, Chugai Pharma, London, UK) to mobilize HPCs into the peripheral blood. Leukapheresis was performed after 4 days of G-CSF treatment.

CD34+ cells were isolated from the apheresis unit using a human CD34 microbead kit (Miltenyi Biotec, Bergisch Gladbach, Germany), according to the manufacturer's instructions. Briefly, $2 \times 10^{8}$ to $4 \times 10^{8}$ mononuclear cells were resuspended in phosphate-buffered saline (PBS) supplemented with $0.5 \%$ bovine serum albumin and incubated with human immunoglobulin (Ig)G (blocking reagent) and human CD34 antibody-coated MACS microbeads. The cells were then washed and loaded onto a magnetic separation column ( $\mathrm{LS}^{+}$column) followed by two washing steps with $3 \mathrm{~mL}$ of PBS to remove unlabeled cells. CD34+ cells were eluted from the column using $5 \mathrm{~mL}$ of elution buffer and washed in PBS before culturing. The CD34+-enriched cell population was cultured for 24 hours in Iscove's modified Dulbecco's medium (IMDM) supplemented with $5 \%$ human $A B$ serum ( $\mathrm{vol} / \mathrm{vol})$, stem cell factor (SCF; $100 \mathrm{ng} / \mathrm{mL}$ ), Fms-like tyrosine kinase 3-ligand (Flt3-L; $100 \mathrm{ng} / \mathrm{mL}$ ), thrombopoietin (TPO; $50 \mathrm{ng} / \mathrm{mL}$ ), and interleukin (IL)-6 (50 ng/mL).

\section{Lentiviral constructs and vector production}

A lentiviral vector was used for stable short-hairpin RNA (shRNA) expression. shRNA expression cassettes (Table 1) were cloned into the lentiviral expression vector $\mathrm{pLVTHm} / \mathrm{si}$ containing enhanced green fluorescent protein (eGFP) as the reporter gene. Lentiviral particles for $\beta 2$-microglobulin $(\beta 2 \mathrm{~m})$ silencing were produced by transfecting $5 \times 10^{6}$ HEK293T cells with $10 \mu$ g of plasmid encoding for $\beta 2 \mathrm{~m}$-specific shRNA, $9 \mu \mathrm{g}$ of plasmid psPAX2 containing gag and pol proteins, and $3 \mu \mathrm{g}$ of pMD2G encoding for VSV-G protein. pLVTHm/si, psPAX2, and PMD2G were provided by D. Trono (Lausanne, Switzerland). A vector encoding a nonsense shRNA (shNS) was used as control. After 16 hours, the cells were washed and incubated with complete Dulbecco's modified Eagle's medium supplemented with $1 \%$ sodium pyruvate (C.C. Pro, Neustadt, Germany). After 24 hours, the lentiviral vector containing supernatant was collected, filtered $(0.45 \mu \mathrm{m})$, and concentrated overnight by centrifugation

\begin{tabular}{|c|c|}
\hline \multicolumn{2}{|c|}{$\begin{array}{l}\text { TABLE 1. Schematic representation of the shRNA } \\
\text { sequence targeting } \beta 2 \mathrm{~m} \text { transcripts (sh } \beta 2 \mathrm{~m})\end{array}$} \\
\hline shRNA sequence & \\
\hline 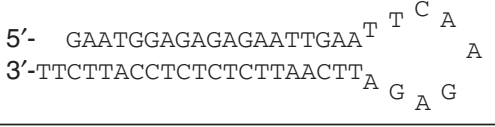 & $\operatorname{sh} \beta 2 m$ \\
\hline
\end{tabular}


at $22,000 \times g$. The virus pellet was resuspended in complete medium.

\section{Lentiviral vector transduction}

Relative infectious titers of the lentiviral vectors were calculated by quantifying the percentage of eGFP-positive cells in HeLa cells transduced with diluted volumes of the concentrated viral supernatants. CD34+ cells $\left(4 \times 10^{5} /\right.$ well) were seeded in fibronectin (RetroNectin, TAKARA Bio Inc., Shiga, Japan)-coated 24-well plate and infected with a multiplicity of infection of 10 in the presence of $8 \mu \mathrm{g} / \mathrm{mL}$ protamine sulfate (Sigma-Aldrich, Steinheim, Germany), SCF (100 ng/mL), Flt3-L (100 ng/mL), IL-6 (50 ng/mL), and TPO (100 ng/mL). After 8 hours, the CD34+ cells were washed using fresh complete medium. Transduction efficiency was calculated by considering the percentage of GFP-expressing cells.

\section{Suspension cultures for the generation of PLTs}

After transduction, $5 \times 10^{4}$ nontransduced, shNS-expressing progenitor cells or HLA Class I silenced progenitor cells, respectively, were cultured in IMDM supplemented with $5 \% \mathrm{AB}$ serum, $20 \mathrm{ng} / \mathrm{mL}$ Flt3-L, $50 \mathrm{ng} / \mathrm{mL}$ TPO, and $50 \mathrm{ng} / \mathrm{mL}$ IL-3 for 5 days. CD34+ progenitor cells expressing eGFP+ as a marker for successful transduction were isolated by a flow cytometry-based cell sorting system (FACSAria, BD Bioscience, San Jose, CA). The GFP-positive cells were then maintained in culture in the presence of $50 \mathrm{ng} / \mathrm{mL}$ TPO and $50 \mathrm{ng} / \mathrm{mL}$ IL-3 for 16 days to allow the differentiation into megakaryocytes and the release of PLTs. ${ }^{9}$

\section{Morphologic analyses of megakaryocytes}

Megakaryocytes expanded in culture were identified with a fluorescence microscope (Keyence, Tokyo, Japan) $3,6,12,17$, and 21 days after transduction, based on shape and adherence properties.

\section{Characterization of megakaryocytic lineage using anti-CD41}

Five days after transduction, cells were collected and centrifuged at $450 \times g$ for 5 minutes and resuspended in PBS. Megakaryocytic lineage cells were iden(20x). tified and quantified by flow cytometry analysis (FACSCanto, BD Bioscience Immunocytometry Systems) after staining with phycoerythrin (PE)-conjugated anti-CD41 (glycoprotein [GP]IIb-IIIa; Serotec, Dusseldorf, Germany; and BD PharMingen, Heidelberg, Germany). A PEconjugated IgG was used as isotype control (BD PharMingen). The data were processed using software (FACSDiva, BD Bioscience Immunocytometry Systems). Two variables, the percentage of antigen-expressing cells and the mean fluorescence intensity as a relative measure for the amount of antigen expression on the cell surface, were used to describe the expression results.

\section{Quantification of PLTs derived from CD34+ progenitor cells}

Twenty-one days after transduction, PLT-containing supernatant was collected and centrifuged at $201 \times g$ to
A.

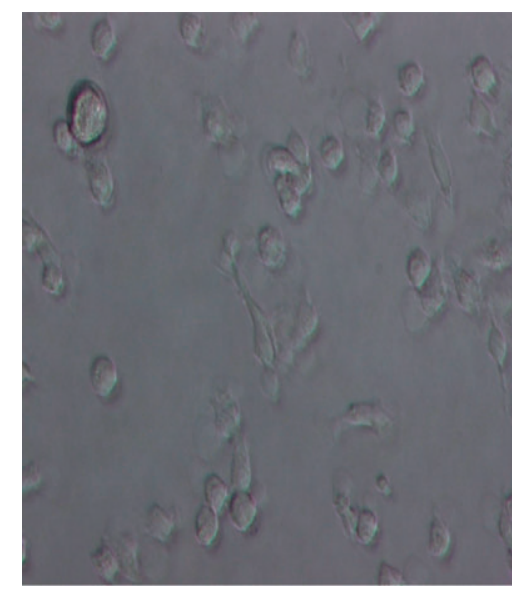

C.

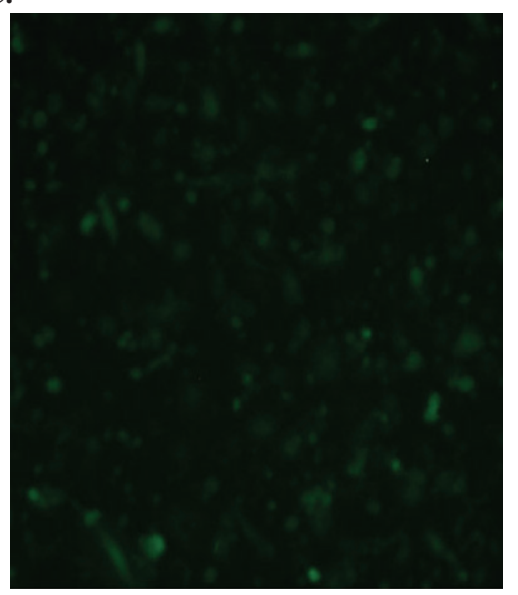

B.

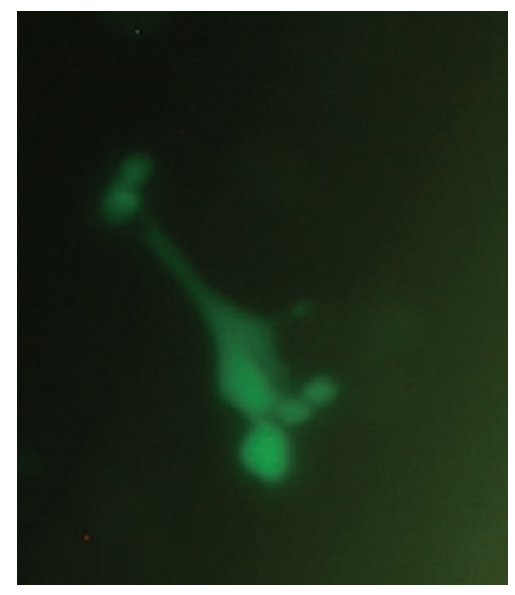

Fig. 1. Microscopic analysis of cell morphology and GFP expression. Representative microscopic images of (A) megakaryocytes, 6 days after transduction (40X); (B) a GFP-expressing, pro-PLT-bearing megakaryocyte, 6 days after transduction (100×); and (C) GFP-expressing progenitor cells expressing $\operatorname{sh} \beta 2 \mathrm{~m} 3$ days after transduction 
remove large cells. The supernatant was further centrifuged at $1800 \times g$ for 5 minutes to obtain a PLT pellet. The PLTs were resuspended in PBS, labeled with PE-conjugated anti-CD61 (GPIIIa) (Serotec) or antiCD42a (GPIX; BD PharMingen), and measured by flow cytometry. The PLT gate was fixed in the forward scatter (FSC) and side scatter (SSC) profiles of blood-derived PLTs from healthy volunteers.

\section{Analysis of HLA Class I expression}

An allophycocyanin-labeled W6/32 antibody (Serotec) that recognizes an antigenic determinant shared among all HLA-A, -B, and -C proteins was used to detect the expression of HLA on the cell surface by flow cytometric analysis. A gate was fixed in the FSC and SSC dot plot with the use of peripheral blood PLTs from healthy donors.

Total RNA was isolated from cells derived from the CD34+ progenitor cells (RNeasy mini kit, Qiagen, Hilden, Germany) 1 and 2 weeks after transduction. The isolated RNA was then reverse transcribed to cDNA using the high-capacity cDNA reverse transcription kit (Applied Biosystems, Darmstadt, Germany). Real-time polymerase chain reaction (PCR) for $\beta 2 \mathrm{~m}$ was performed using the oligonucleotide pairs and MGB-TaqMan probes as described elsewhere. ${ }^{16}$ The thermal cycling conditions used on a real-time PCR system (ONEStep Plus, Applied Biosystems) were $50^{\circ} \mathrm{C}$ for 15 minutes and $95^{\circ} \mathrm{C}$ for 10 minutes followed by 40 cycles at $95^{\circ} \mathrm{C}$ for 15 seconds and $60^{\circ} \mathrm{C}$ for 1 minute. The constitutively expressed $\beta$-actin gene was amplified as the reference standard for normalization of mRNA levels. All real-time PCR analyses were performed in triplicate.

\section{PLT activation assay with adenosine 5'-diphosphate and thrombin}

PLTs generated from CD34+ progenitor cells or blood-derived PLTs as controls were stimulated with $25 \mu \mathrm{mol} / \mathrm{L}$ adenosine $5^{\prime}$-diphosphate (ADP) plus $1 \mathrm{U}$ of thrombin for 10 minutes and immediately fixed with fixation solution (Cytofix, BD PharMingen). To assess PLT activation by flow cytometry, PLTs were stained with PE-conjugated anti-CD62P (P-selectin, BD PharMingen) or purified anti-activated GPIIb-IIIa antibody (PAC-1, BD PharMingen) for 30 minutes.
In the latter case, a PE-conjugated anti-mouse IgG (BD PharMingen) was used as the detection antibody.

\section{PLT aggregation assay after ADP and thrombin stimulation}

A mean of $1.8 \times 10^{6}$ PLTs generated from CD34+ progenitor cells or blood-derived PLTs suspended in $100 \mu \mathrm{L}$ of PBS were incubated with $40 \mu \mathrm{mol} / \mathrm{L}$ ADP plus $1 \mathrm{U}$ thrombin for 10 minutes. Aggregation was then analyzed by flow cytometry analysis. In the stimulated PLTs, particles with higher FSC and SSC are observed, indicating aggregate formation.

\section{PLT aggregation assay after collagen stimulation}

A mean of $2 \times 10^{7}$ PLTs generated from CD34+ progenitor cells or blood-derived PLTs resuspended in $200 \mu \mathrm{L}$ of plasma were stimulated with $10 \mu \mathrm{g}$ collagen (Nycomed, Linz, Austria). Aggregation was measured using an

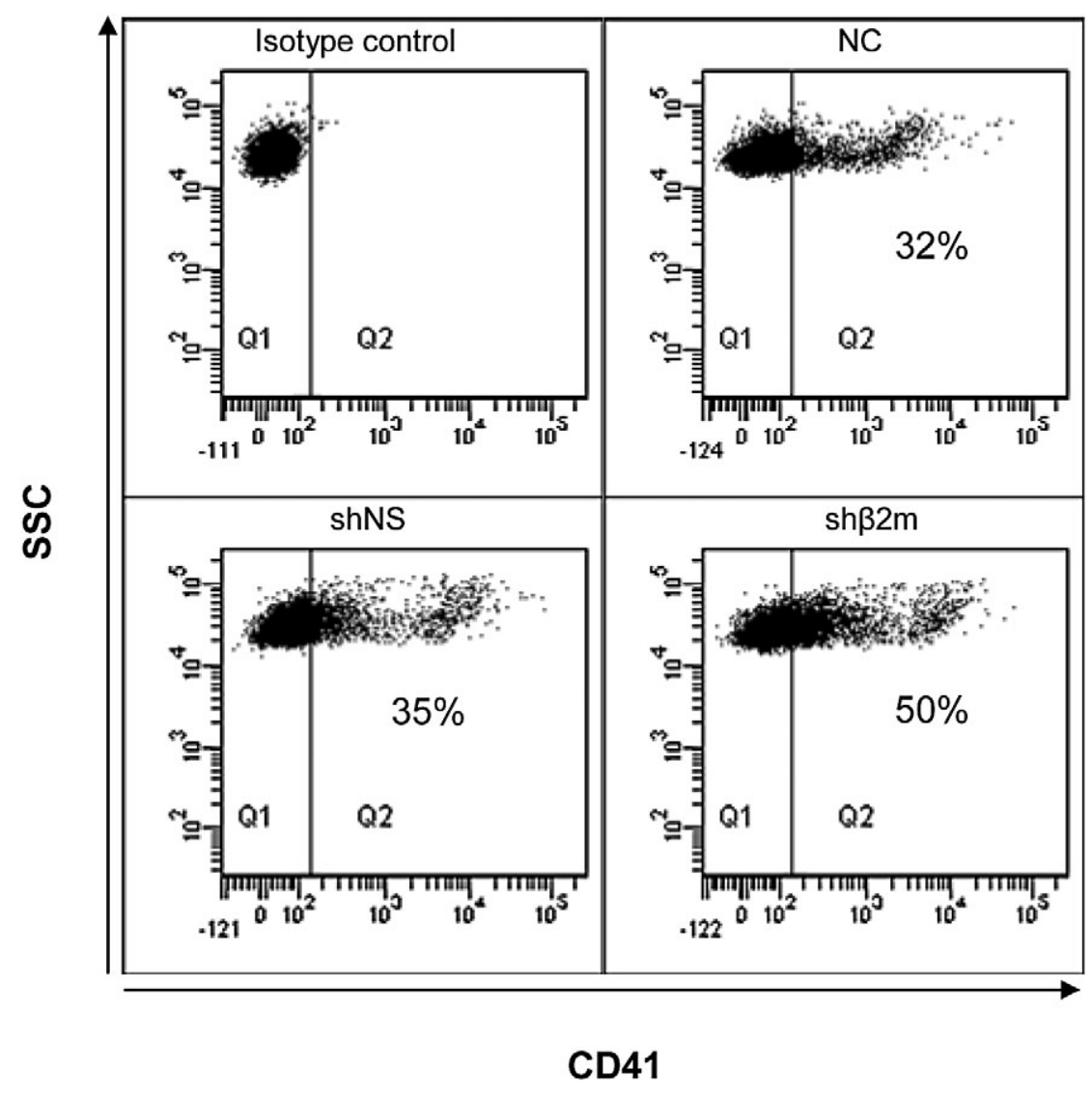

Fig. 2. CD41 expression on CD34+ progenitor-derived megakaryocytes. CD34+ progenitor cells were cultured in the presence of TPO and IL-3. CD41 expression on nontransduced cells (negative control [NC]), megakaryocytes derived from progenitor cells transduced with the vector encoding for shNS, and megakaryocytes derived from progenitor cells transduced with the vector encoding for $\operatorname{sh} \beta 2 \mathrm{~m}$ was measured by flow cytometry after 5 days of culture. 
aggregometer (PAP4, Bio/Data Corp., Horsham, PA) 30 seconds and 5 minutes after collagen addition.

\section{RESULTS}

\section{Generation of genetically modified megakaryocytes and PLTs from CD34+ progenitor cells}

Megakaryocytes were identified based on their morphology and CD41 (GPIIb-IIIa) expression. There was no difference in the phenotype of the progenitor cell-derived megakaryocytes between the cultures in which transduced and those in which nontransduced CD34+ cells were used for expansion and megakaryocyte lineage differentiation (Fig. 1A). The megakaryocytes exhibited long beaded projections, so-called pro-PLTs, from which PLTs were produced (Fig. 1B). ${ }^{17}$ There were no differences in the proportions of generated megakaryocytes between the cultures based on transduced and those based on nontransduced CD34+ cells. Five days after transduction, the proportions of CD41+ cells ranged from $25 \%$ to $53 \%$ (mean, 40\%-42\%; Fig. 2). Megakaryoctes yields tended to be higher for CD34+ progenitor cells transduced with shRNA-encoding vectors than for the nontransduced progenitor cells, although the differences were not significant. Interestingly, the highest number of megakaryocytes were obtained from the progenitor cells that had been treated with vectors encoding for $\beta 2 \mathrm{~m}$-specific shRNAs: after 1 week of culture in the presence of TPO and IL-3 for PLT differentiation, $5 \times 10^{4} \mathrm{CD} 34+$ progenitor cells produced a mean of $25.7 \times 10^{3} \pm 11.2 \times 10^{3} \mathrm{CD} 41+$ cells (Fig. 3A).

PLTs were identified based on the scatter properties of peripheral blood PLTs from healthy donors and the PLTspecific antigens CD61 (GPIIIa) and CD42a (GPIX). The first PLTs were observed around Day 8 of cell culture. Flow cytometry analysis verified that culture-derived PLTs exhibited a similar but broader size distribution than freshly isolated blood PLTs (data not shown). ${ }^{18}$ After 21 days, the mean percentages of $\mathrm{CD} 34+$ progenitor cellderived PLTs were similar under different treatment conditions, ranging from 55 to $65 \%$ when CD61 was used as the marker for PLT identification and from 25 to $30 \%$ when CD42a was used (Fig. 4). The imbalance in CD61 and CD42a expression on progenitor cell-derived PLTs compared to the expression profile of peripheral bloodderived PLTs suggests that the culture protocol used in this study may not completely mimic the natural conditions in the marrow. ${ }^{18}$ According to the megakaryocyte yield results, cultures with CD34+ cells treated to express $\beta 2 \mathrm{~m}$ specific shRNAs tended to produce larger numbers of PLTs than the cultures with nontransduced progenitor cells or progenitor cells expressing nonspecific shRNAs (Fig. 3B). On Day 21 of cultivation the concentration of CD61+ PLTs
A.

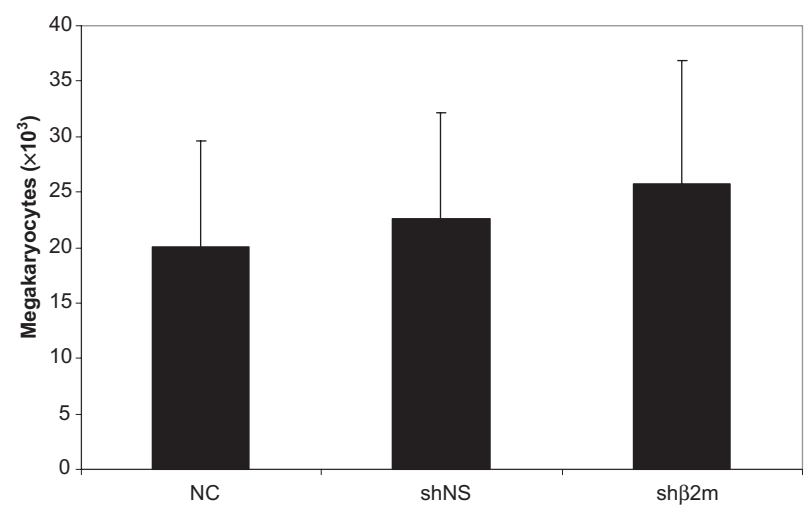

B.

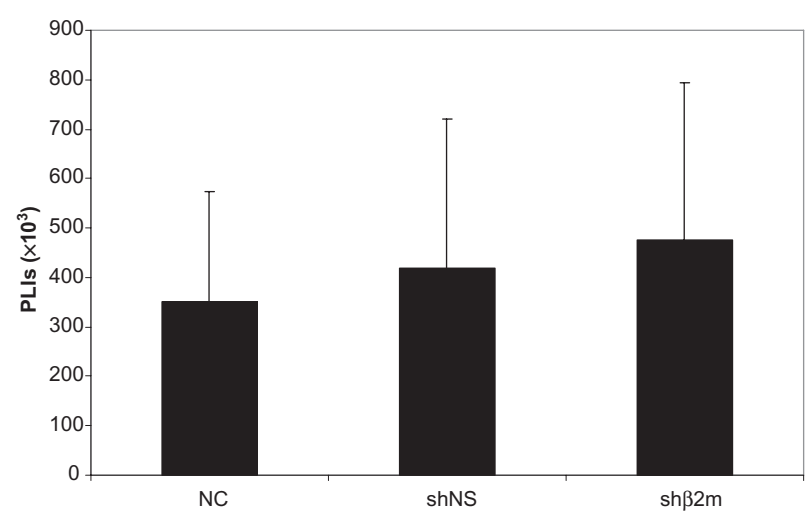

Fig. 3. Quantification of CD34+ progenitor cell-derived megakaryocytes and PLTs. Megakaryocytes (A) and PLTs (B) stained with anti-CD41 and anti-CD61, respectively, were counted by flow cytometry after 21 days of cell culture. The CD34+ progenitor cell-derived megakaryocytes and PLTs were harvested for analysis of nontransduced cell cultures (negative control [NC]), cell cultures treated with the vector encoding for shNS, and cell cultures transduced with the vector encoding for $\operatorname{sh} \beta 2 \mathrm{~m}$.

released by CD34+-derived megakaryocytes reached up to $475 \times 10^{3} \pm 320 \times 10^{3} / \mathrm{mL}$ of culture.

\section{Silencing of HLA Class I expression}

We constructed a VSV-G pseudotyped lentivirus vectors for the stable delivery of a $\beta 2 \mathrm{~m}$-specific shRNA or a nonspecific shRNA as control. These vectors also encoded for eGFP as reporter gene. A mean of $35 \pm 20 \%$ GFPexpressing cells was obtained after transduction of the CD34+ progenitor cells (Fig. 1C). Five days after transduction, real-time PCR showed up to $95 \%$ reduction of $\beta 2 \mathrm{~m}$ mRNA levels in progenitor cells treated for shRNAs specific for $\beta 2 \mathrm{~m}$ ( $\operatorname{sh} \beta 2 \mathrm{~m}$ ) expression compared to levels in nontransduced cells or those expressing the nonspecific shRNA (Fig. 5A). Accordingly, a reduction in HLA Class I 
A.

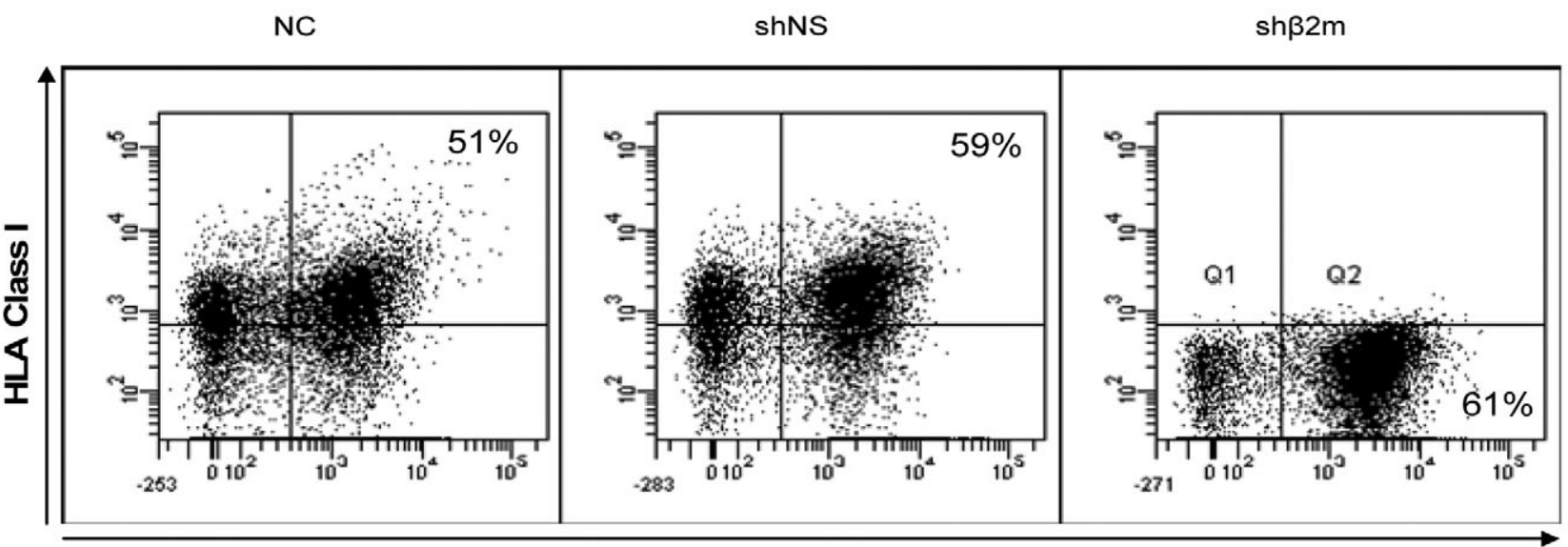

B.
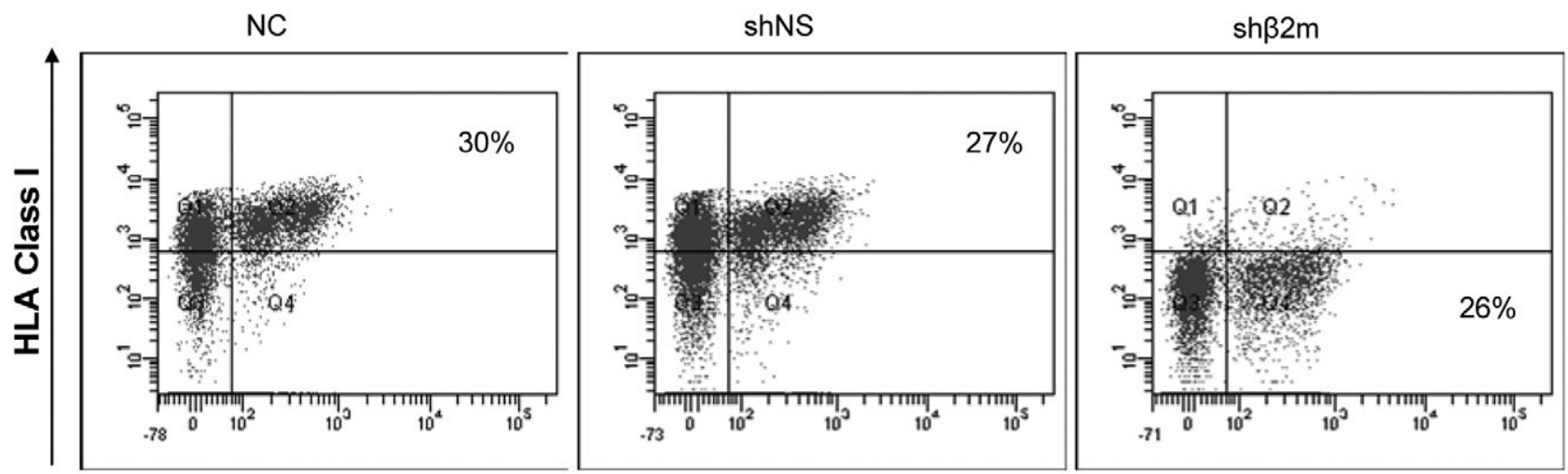

CD42a

Fig. 4. Expression of CD61 and CD42a PLT-specific antigens on CD34+ progenitor cell-derived PLTs. After 21 days of culture, PLTs derived from the CD34+ progenitor cells were harvested and the expression of CD61 (A) and CD42a (B) was measured by flow cytometry on PLTs derived from nontransduced progenitor cells (negative control [NC]), PLTs derived from progenitor cells transduced with the vector encoding for shNS, or PLTs derived from progenitor cells transduced with the vector encoding for sh $\beta 2 \mathrm{~m}$.

protein expression by up to $85 \%$ was observed in the CD34+ cells expressing the $\beta 2 \mathrm{~m}$-specific shRNAs compared to those cells that had been transduced with the control construct (Fig. 5B). Up to 85\% HLA Class I antigen reduction was observed in PLTs that derived from $\beta 2 \mathrm{~m}$ specific shRNAs expressing progenitor cells, suggesting that RNAi-mediated HLA deficiency was maintained during PLT maturation (Fig. 4). The degree of HLA Class I silencing on PLTs remained stable during the entire culture period (data not shown).

\section{Functional assessment of HLA Class I-silenced PLTs}

To explore the functionality of CD34+ progenitor cellderived HLA Class I-deficient PLTs derived from, we used flow cytometry to perform PLT aggregation analyses and to examine GPIIb-IIIa activation and CD62P (P-selectin) antigen up regulation on PLTs after stimulation with the major PLT agonists thrombin and ADP. The expression of activated GPIIb-IIIa and CD62P on the CD34+ progenitor cell-derived PLTs was clearly evoked by this treatment in a fashion similar to those from healthy volunteers (Fig. 6). In particular, there was no difference in ADP- and thrombin-induced activation between the HLA Class I-silenced and the nonsilenced PLTs. A mean of $75 \%$ of HLA Class I-silenced PLTs up regulated CD62P expression and the expression of activated GPIIb-IIIa was increased by up to $35 \%$ on these PLTs. Activation with ADP and thrombin produced similar values in PLTs derived from both nontransduced CD34+ progenitor cells and PLTs generated from progenitor cells expressing nonspecific shRNAs (CD62P up regulation of $70 \pm 27 \%$ and PAC- 1 binding of $30 \pm 17 \%$ ). Blood-derived PLTs exhibited CD62P expression levels of $82 \pm 17 \%$ and PAC- 1 binding of $26 \pm 7 \%$ after stimulation. 
Our aggregation analysis also suggested that HLA Class I-silenced PLTs derived from HPCs were as functional as nonsilenced PLTs and PLTs from peripheral blood. There was no difference in thrombin-induced aggregation in these different types of PLTs (Fig. 7A). PLT aggregation was detectable in $36 \pm 25 \%$ of the HLAsilenced PLTs. After stimulation, $35 \pm 26 \%$ of nontransduced progenitor cell-derived PLTs and $30 \pm 22 \%$ of shNS-expressing progenitor cell-derived PLTs formed aggregates. Similarly, stimulation of blood-derived PLTs

A.

NC
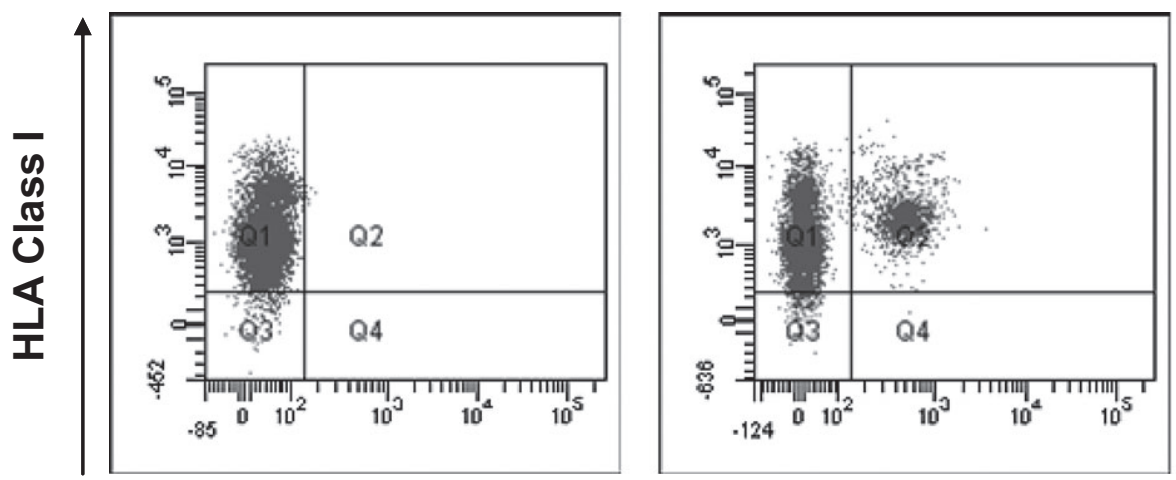

$\operatorname{sh} \beta 2 m$

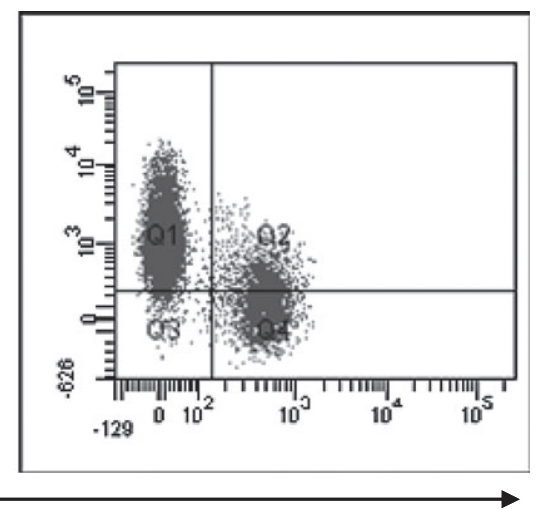

\section{GFP}

B.

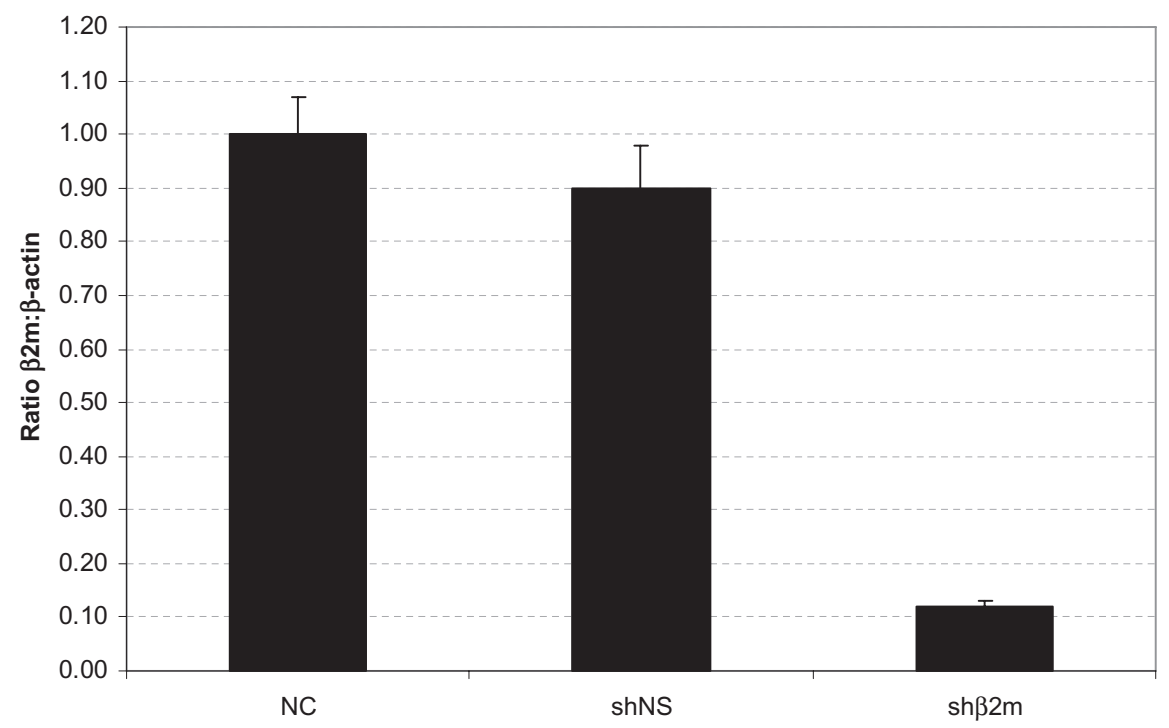

Fig. 5. Silencing of HLA Class I expression in CD34+ progenitor cells. A lentiviral-based vector was used for the delivery of shRNAs specific for $\beta 2 \mathrm{~m}$ or nonspecific shRNAs. (A) HLA Class I surface expression and (B) $\beta 2 \mathrm{~m}$ transcript levels were measured by flow cytometry and real-time PCR, respectively, in nontransduced progenitor cells (negative control [NC]), progenitor cells transduced with the vector encoding for shNS, and progenitor cells transduced with the vector encoding for $\operatorname{sh} \beta 2 \mathrm{~m} \mathbf{5}$ days after transduction and GFP-positive cell sorting.

Fig. 6. Assessment of activated GPIIb-IIIa and CD62P (P-selectin) expression on CD34+ progenitor cell-derived PLTs after stimulation. Activated GPIIb-IIIa (A) and CD62P (B) were measured in the absence and presence of thrombin (25 $\mu \mathrm{mol} / \mathrm{L})$ and ADP (1 U) on PLTs derived from blood, progenitor cells transduced with the vector encoding for shNS, and progenitor cells transduced with the vector encoding for $\operatorname{sh} \beta 2 \mathrm{~m}$. For assessment of activated GPIIb-IIIa the cells were stained with PAC-1, an antibody that mimics the specific fibrinogen binding to human GPIIb-IIIa in the activated state. 
A.
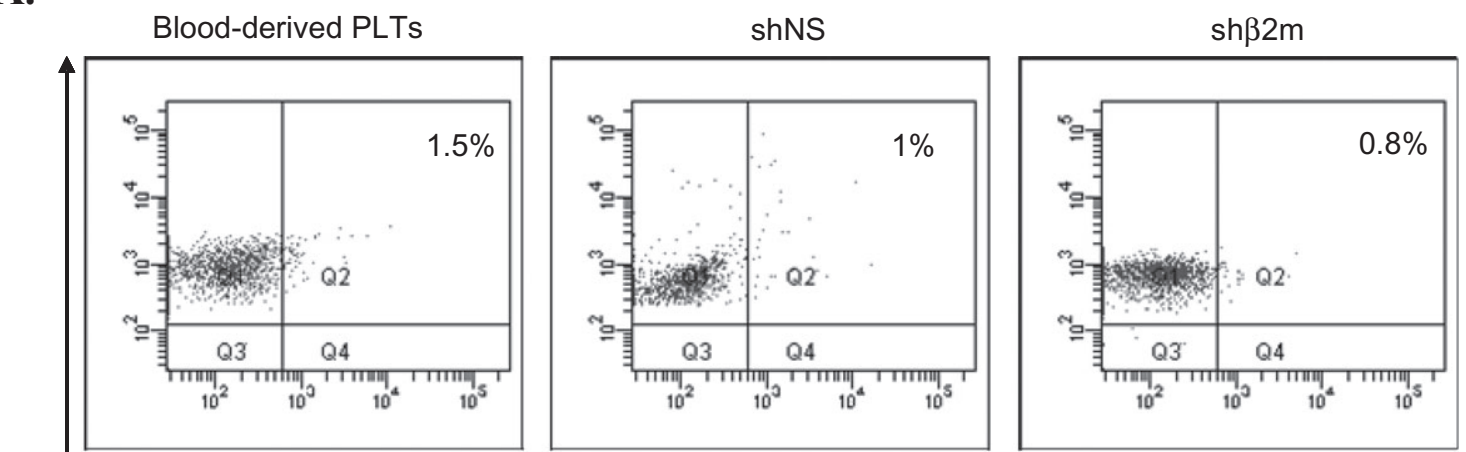

Nonstimulated

œ
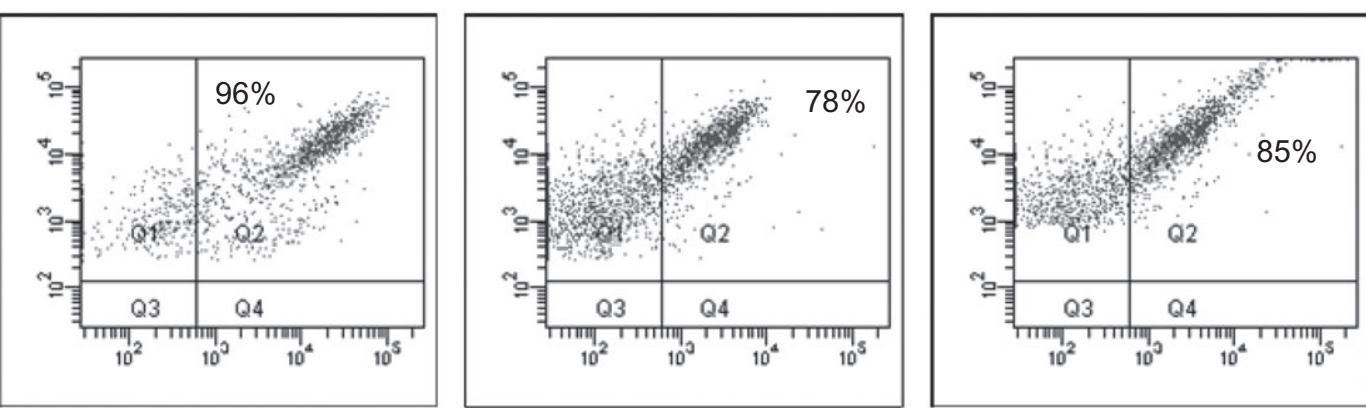

Stimulated

\section{CD62P}

B.
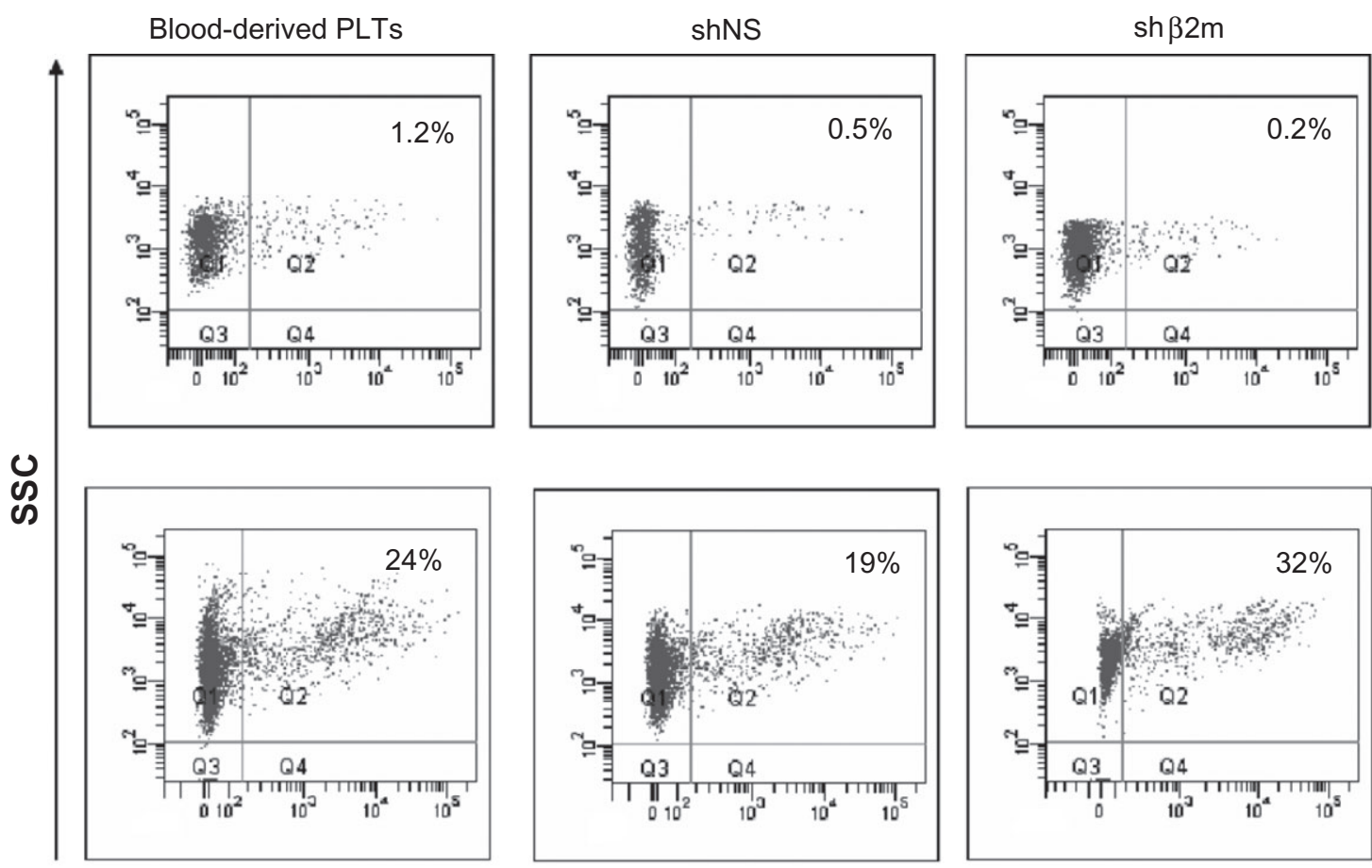

Nonstimulated
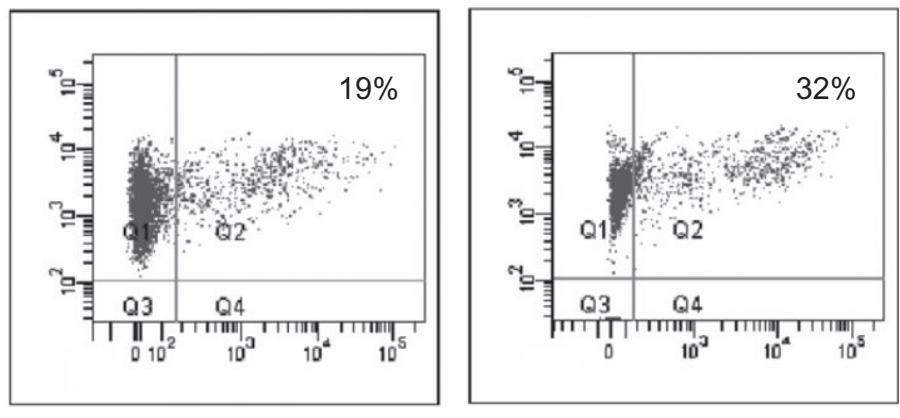

Stimulated

PAC-1 
Nonstimulated
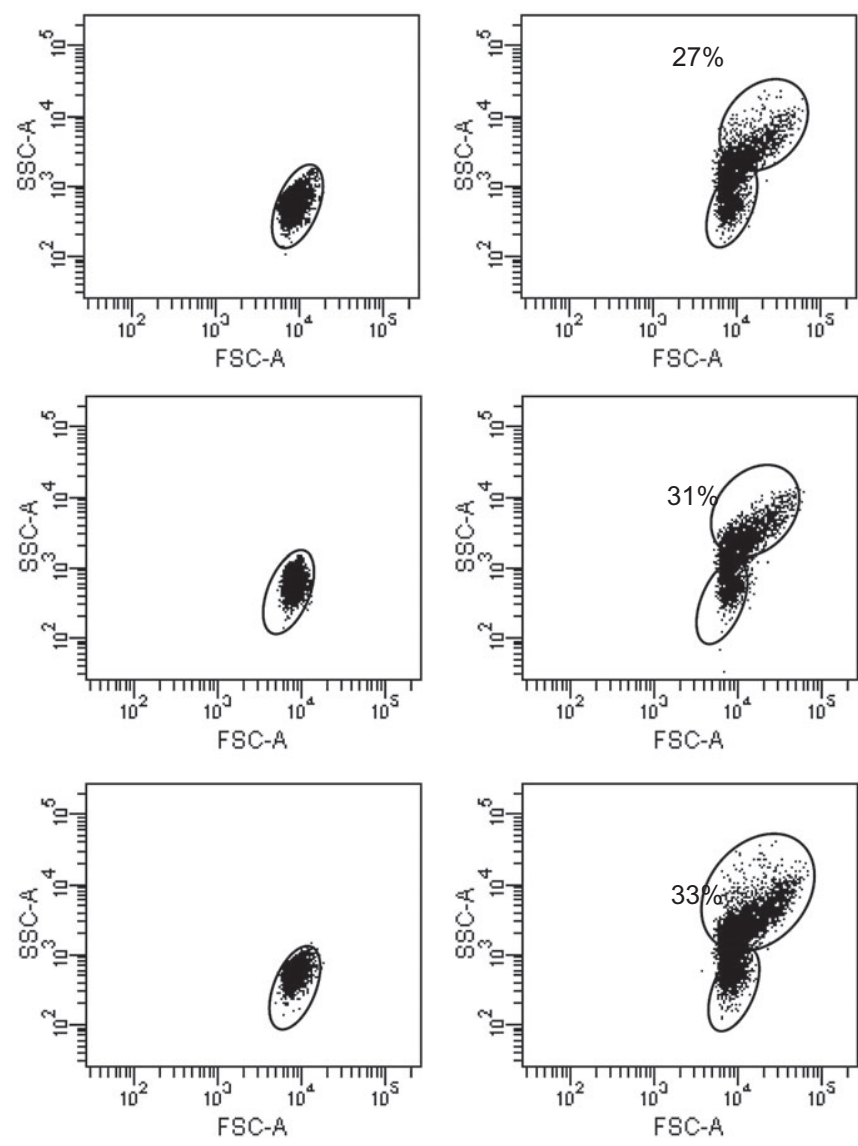

B.

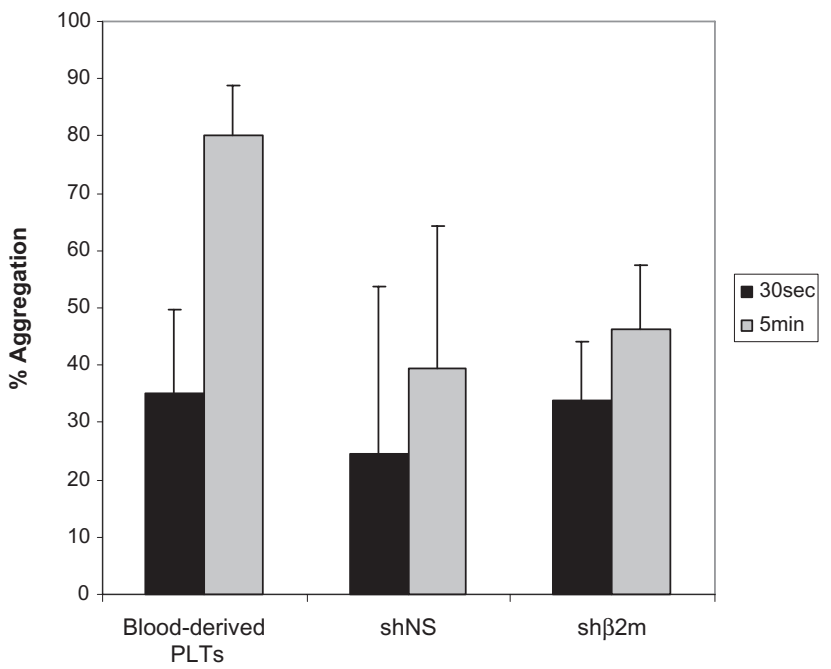

Fig. 7. PLT aggregation assay. (A) PLTs isolated from peripheral blood, PLTs derived from CD34+ progenitor cells expressing shNS, or HLA Class I-silenced PLTs derived from progenitor cells expressing $\operatorname{sh} \beta 2 \mathrm{~m}$ were incubated in the absence or presence of ADP $(40 \mu \mathrm{mol} / \mathrm{L})$ and thrombin ( $1 \mathrm{U})$ for 10 minutes. PLT aggregation was identified based on a shift in the FSC and SSC properties. The percentage of PLT aggregation upon stimulation is indicated in each dot plot. (B) PLTs derived from blood or differentiated from CD34+ cells expressing $\operatorname{shNS}$ or $\operatorname{sh} \beta 2 \mathrm{~m}$ were stimulated with $10 \mu \mathrm{g}$ of collagen, and PLT aggregation was measured with a PAP4 aggregometer. The graph represents a mean of five independent experiments. resulted in aggregation of $42 \pm 21 \%$ of the PLT population. In response to collagen, PLTs isolated from peripheral blood showed a mean of aggregation of $35 \pm 8 \%$ and $80 \pm 10 \% 30$ seconds and 5 minutes, respectively, upon stimulation (Fig. 7B). Thirty seconds and 5 minutes after collagen stimulation, $24 \pm 29$ and $39 \pm 24.9 \%$ of the PLTs derived from CD34+ progenitor cells expressing shNS were able to aggregate. PLT aggregation was detectable in $33 \pm 11 \%$ and $46 \pm 9 \%$ of the HLA Class I-silenced PLTs 30 seconds and 5 minutes, respectively, after collagen stimulation. For interpretation of the PLT aggregation results obtained by the PAP4 aggregometer it must be taken into account that due to their limited availability the cell culture-derived PLTs could not be used in the concentrations this assay has originally been validated for.

\section{DISCUSSION}

In recent years, an increasing number of studies reported culture methods for generation of human blood cells from progenitor cells. Functional red blood cells (RBCs) and PLTs were obtained from hematopoietic stem cells of diverse origins: peripheral blood, marrow, and cord blood..$^{8,9,18-23}$ In addition, embryonic stem cells with their unlimited proliferative potential as well as induced pluripotent stem cells have been considered as a new source for in vitro hematopoiesis; culture systems have been developed that enable embryonic stem cells to efficiently develop into hematopoietic progenitors capable of releasing functional blood cells. $^{10-12,24,25}$ The degree of large-scale expansion and maturation achieved appears very promising for the ex vivo generation of PLT and RBC units for clinical applications. Such methods of generating blood cells from embryonic stem cells or pluripotent stem cells could make it possible to produce a constant and safe supply of blood cells, thus eliminating the need to obtain blood cells from blood donation. This perspective has motivated research groups around the world as well as the US mili- 
tary to launch "blood pharming" programs aimed at creating systems to produce RBCs derived from progenitor cells for use in blood transfusions.

The concept of using progenitor cells for mass production of blood cells holds the potential to genetically manipulate the starting material to produce designer blood cells for multiply transfused patients for whom it is difficult to resource compatible blood. Analogously to what has recently been shown for cell culture-derived $\mathrm{RBCs},{ }^{26}$ we were able to generate in vitro human PLTs with a target phenotype different from the original donor phenotype. We used a combination of lentiviral gene transfer and RNAi technology to silence HLA Class I expression in PLTs derived from CD34+ cells. The dimeric structure of the HLA Class I molecule, comprising the polymorphic HLA heavy chain and the conserved $\beta 2 \mathrm{~m}$ molecule, was used to develop the strategy for Class I-specific HLA silencing. Target sequences within the coding region of $\beta 2 \mathrm{~m}$ were selected to design shRNAs to silence all HLA Class I molecules on the cell surface. Stable reduction of HLA Class I antigen expression by up to $85 \%$ on progenitor cells and PLTs suggests that the expression of shRNA in hematopoietic stem cells was efficient and maintained during megakaryocyte differentiation, thus resulting in inhibition of targeted protein in PLTs. Moreover, the silencing strategy seemed to be satisfactorily efficient to inhibit de novo synthesis of HLA Class I molecules from residual mRNA in mature PLTs during storage in the culture medium. These data indicate that lentiviralmediated RNAi is a powerful tool for continuous production of genetically engineered PLTs.

The idea of providing HLA-reduced PLTs for heavily HLA Class I-alloimmunized patients is not new. Elution techniques with chloroquine or citric acid have been used to remove HLA antigens from PLTs. The first successful transfusion of HLA-eluted PLTs in an HLA-alloimmunized patient was reported by Shanwell and colleagues in $1991 .^{27}$ They demonstrated that the degree of HLA reduction varies, which raised doubt about the application of HLAeluted PLTs for transfusion purposes. ${ }^{28}$ Further progress was made toward the standardization of HLA reduction by acid elution and although acid-treated PLT transfusions were successfully used on a small scale, ${ }^{29-31}$ PLT quality could become critically affected by the PLT preparation procedure. However, even if the clinical and in vivo survival data obtained with acid-treated PLTs are only based on case studies, they suggest that HLA-deficient PLTs may survive almost normally in the circulation and be functionally intact. This is to be expected in particular because HLA reduction on silenced PLTs (up to 85\%) was similar to what was described for acid-treated PLTs and silenced PLTs are not subjected to any harsh preparation procedures. In previous studies, we could demonstrate in microcytotoxicity tests that RNAi-mediated silencing of the HLA Class I expression prevented antibody-mediated cytotoxicity ${ }^{16,32}$ In addition, T-cell cytotoxicity and proliferation showed to be significantly reduced when allogeneic $\mathrm{T}$ cells were exposed to HLA-deficient target cells. Our observation that HLA Class I antigen expression on HLA-silenced PLTs was not up regulated after in vitro stimulation suggests that the posttransfusion increment in HLA-alloimmunized patients may not be impaired by de novo synthesis of HLA on activated PLTs. Nevertheless, studies in animals and humans are needed to determine the success rate and tolerability of HLA-silenced, culturederived PLTs.

The functional role of HLA Class I on PLTs is not entirely clear. In blocking studies, Cosgrove and coworkers $^{33}$ showed that both heavy and light chains of HLA Class I antigens might be involved in PLT aggregation and release reactions. HLA alloantibodies could induce the aggregation of human PLTs and selectively block the binding of some PLT agonists (e.g., collagen and adrenalin) but not of others (e.g., thrombin). Rubinstein and colleagues ${ }^{34}$ suggested that HLA Class I might synergize with the Fc $\gamma$ RII receptor to activate PLTs. Although a specific function of PLT membrane HLA antigens cannot be excluded, our in vitro data suggest that expression of PLTspecific membrane GPs and PLT function are not affected in HLA-silenced PLTs. The silencing strategy presented herein might be an attractive tool to be used in an autologous animal model to further determine the functional relevance of HLA Class I on PLTs.

Important safety concerns exist whenever gene transfer is used to manipulate progenitor cells for clinical purposes. ${ }^{35-37}$ The main difference to be considered in risk assessments between clinical trials using genetically engineered progenitor cells for transplant purposes itself or PLTs derived thereof in cultures for transfusion purposes is the complete absence of any proliferation potential of fully maturated PLTs. This virtually eliminates any risk of malignant transformation of transfused PLTs making the development of leukemia in the recipient improbable. Moreover, standard blood preparation techniques are available to ensure that transfusion units contain only maturated PLTs by eliminating or inactivating contaminating nucleated cells: leukoreduction filters could be used to effectively eliminate any contaminating undifferentiated progenitor cells and PLTs, as anucleate fragments of cytoplasm, can easily be irradiated before transfusion.

In conclusion, this study shows for the first time the feasibility of generating functional HLA Class I-deficient PLTs from CD34+ progenitor cells. Blood products containing HLA-deficient PLTs may become a potent tool to overcome clinical refractoriness in multiply transfused patients. At present, transfusion units from large-scale production of cultured blood cells appear to be a technological luxury, considering the costs to establish industrial production conditions compatible with transfusion requirements and the rules of good manufacturing 
practice. For example, current strategies to produce PLTs from HPCs include expensive cytokines continuously to be added to the culture media. However, the new technology presented here makes it possible to produce designer blood products that can be live-saving in certain clinical situations. The provision of specially designed blood units such as HLA-silenced PLT concentrates may help to improve the cost-benefit ratio of in vitro generated blood cells. Currently, the amount of PLTs generated in any experimental setting fails to satisfy the clinically required quantity for transfusion. ${ }^{8,9}$ The key challenge is the efficient and continuous expansion of progenitor cells that can differentiate into mature PLTs. Human embryonic or induced pluripotent stem cells, which have an unlimited proliferative potential, could become in the next years an attractive resource for blood cell engineering. However, the technical conditions for industrial development must still be created. In addition, a better understanding of the megakaryocytosis and the mechanism of PLT production is needed to optimize cell culture conditions.

\section{ACKNOWLEDGMENTS}

The authors thank Jana Zenk for her technical assistance. This study was supported in part by the Deutsche Forschungsgemeinschaft and the Excellence Cluster REBIRTH (DFG SE 1093/2-1, EXC 62).

\section{CONFLICT OF INTEREST}

The authors declare that they have no competing financial interest.

\section{REFERENCES}

1. Gulliksson H, AuBuchon JP, Vesterinen M, Sandgren P, Larsson S, Pickard CA, Herschel I, Roger J, Tracy JE, Langweiler M; Biomedical Excellence for Safer Transfusion Working Party of the International Society of Blood Transfusion. Storage of platelets in additive solutions: a pilot in vitro study of the effects of potassium and magnesium. Vox Sang 2002;82:131-6.

2. Blumberg N, Heal JM, Liesveld JL, Phillips GL, Rowe JM. Platelet transfusion and survival in adults with acute leukemia. Leukemia 2007;22:631-5.

3. Nevo S, Fuller AK, Zahurak ML, Hartley E, Borinsky ME, Vogelsang GB. Profound thrombocytopenia and survival of hematopoietic stem cell transplant patients without clinically significant bleeding, using prophylactic platelet transfusion triggers of $10 \times 10(9)$ or $20 \times 10(9)$ per L. Transfusion 2007;47:1700-9.

4. Stephan F, Montblanc J, Cheffi A, Bonnet F. Thrombocytopenia in critically ill surgical patients: a case-control study evaluating attributable mortality and transfusion requirements. Crit Care 1999;3:151-8.

5. Rebulla P. Refractoriness to platelet transfusion. Curr Opin Hematol 2002;9:516-20.

6. Kiefel V, Konig C, Kroll H, Santoso S. Platelet alloantibodies in transfused patients. Transfusion 2001;41:766-70.

7. Laundy GJ, Bradley BA, Rees BM, Younie M, Hows JM. Incidence and specificity of HLA antibodies in multitransfused patients with acquired aplastic anemia. Transfusion 2004; 44:814-25.

8. Matsunaga T, Tanaka I, Kobune M, Kawano Y, Tanaka M, Kuribayashi K, Iyama S, Sato T, Sato Y, Takimoto R, Takayama T, Kato J, Ninomiya T, Hamada H, Niitsu Y. Ex vivo large-scale generation of human platelets from cord blood CD34+ cells. Stem Cells 2006;24:2877-87.

9. Norol F, Vitrat N, Cramer E, Guichard J, Burstein SA, Vainchenker W, Debili N. Effects of cytokines on platelet production from blood and marrow CD34+ cells. Blood 1998;91:830-43.

10. Fujimoto TT, Kohata S, Suzuki H, Miyazaki H, Fujimura K. Production of functional platelets by differentiated embryonic stem (ES) cells in vitro. Blood 2003;102:4044-51.

11. Nishikii H, Eto K, Tamura N, Hattori K, Heissig B, Kanaji T, Sawaguchi A, Goto S, Ware J, Nakauchi H. Metalloproteinase regulation improves in vitro generation of efficacious platelets from mouse embryonic stem cells. J Exp Med 2008;205:1917-27.

12. Takayama N, Nishikii H, Usui J, Tsukui H, Sawaguchi A, Hiroyama T, Eto K, Nakauchi H. Generation of functional platelets from human embryonic stem cells in vitro via ES-sacs, VEGF-promoted structures that concentrate hematopoietic progenitors. Blood 2008;111:5298-306.

13. Abbas-Terki T, Blanco-Bose W, Deglon N, Pralong W, Aebischer P. Lentiviral-mediated RNA interference. Hum Gene Ther 2002;13:2197-201.

14. Taulli R, Accornero P, Follenzi A, Mangano T, Morotti A, Scuoppo C, Forni PE, Bersani F, Crepaldi T, Chiarle R, Naldini L, Ponzetto C. RNAi technology and lentiviral delivery as a powerful tool to suppress Tpr-Met-mediated tumorigenesis. Cancer Gene Ther 2005;12:456-63.

15. Kock N, Kasmieh R, Weissleder R, Shah K. Tumor therapy mediated by lentiviral expression of shBcl-2 and S-TRAIL. Neoplasia 2007;9:435-42.

16. Figueiredo C, Seltsam A, Blasczyk R. Class-, gene-, and group-specific HLA silencing by lentiviral shRNA delivery. J Mol Med 2006;84:425-37.

17. Italiano JE, Jr, Lecine P, Shivdasani RA, Hartwig JH. Blood platelets are assembled principally at the ends of proplatelet processes produced by differentiated megakaryocytes. J Cell Biol 1999;147:1299-312.

18. Ungerer M, Peluso M, Gillitzer A, Massberg S, Heinzmann U, Schulz C, Münch G, Gawaz M. Generation of functional culture-derived platelets from CD34+ progenitor cells to study transgenes in the platelet environment. Circ Res 2004;95:e36-44. 
19. Giarratana MC, Kobari L, Lapillonne H, Chalmers D, Kiger L, Cynober T, Marden MC, Wajcman H, Douay L. Ex vivo generation of fully mature human red blood cells from hematopoietic stem cells. Nat Biotechnol 2005;23: 69-74.

20. Baek EJ, Kim HS, Kim S, Jin H, Choi TY, Kim HO. In vitro clinical-grade generation of red blood cells from human umbilical cord blood CD34+ cells. Transfusion 2008;48: 2235-45.

21. Dorn I, Lazar-Karsten P, Boie S, Ribbat J, Hartwig D, Driller B, Kirchner H, Schlenke P. In vitro proliferation and differentiation of human CD34+ cells from peripheral blood into mature red blood cells with two different cell culture systems. Transfusion 2008;48:1122-32.

22. Neildez-Nguyen TM, Wajcman H, Marden MC, Bensidhoum M, Moncollin V, Giarratana MC, Kobari L, Thierry D, Douay L. Human erythroid cells produced ex vivo at large scale differentiate into red blood cells in vivo. Nat Biotechnol 2002;20:467-72.

23. Choi ES, Nichol JL, Hokom MM, Hornkohl AC, Hunt P. Platelets generated in vitro from proplatelet-displaying human megakaryocytes are functional. Blood 1995;85:40213.

24. Lu SJ, Feng Q, Park JS, Vida L, Lee BS, Strausbauch M, Wettstein PJ, Honig GR, Lanza R. Biologic properties and enucleation of red blood cells from human embryonic stem cells. Blood 2008;112:4475-84.

25. Olivier EN, Qiu C, Velho M, Hirsch RE, Bouhassira EE. Large-scale production of embryonic red blood cells from human embryonic stem cells. Exp Hematol 2006;34:163542.

26. Bagnis C, Chapel S, Chiaroni J, Bailly P. A genetic strategy to control expression of human blood group antigens in red blood cells generated in vitro. Transfusion 2009.

27. Shanwell A, Sallander S, Olsson I, Gulliksson H, Pedajas I, Lerner R. An alloimmunized, thrombocytopenic patient successfully transfused with acid-treated, random-donor platelets. Br J Haematol 1991;79:462-5.

28. Bertolini F, Porretti L, Corsini C, Rebulla P, Sirchia G.
Platelet quality and reduction of HLA expression in acid-treated platelet concentrates. Br J Haematol 1993;83:525-7.

29. Novotny VM, Doxiadis IN, van Doorn R, Brand A. The kinetics of HLA Class I elution and the relevance for the use of HLA-eluted platelet transfusions. Br J Haematol 1996;95:416-22.

30. Castro E, Muncunill J, Barea L, Gonzalez R, FernandezVillalta MJ. Acid elution of platelets HLA-Class I antigens in the treatment of a refractory patient. Br J Haematol 1998;100:245-6.

31. Novotny VM, Huizinga TW, van Doorn R, Briet E, Brand A. HLA Class I-eluted platelets as an alternative to HLAmatched platelets. Transfusion 1996;36:438-44.

32. Figueiredo C, Horn PA, Blasczyk R, Seltsam A. Regulating MHC expression for cellular therapeutics. Transfusion 2007;47:18-27.

33. Cosgrove LJ, Vaughan HA, Tjandra JJ, Thurlow PJ, McKenzie IF. HLA (Class I) antigens on platelets are involved in platelet function. Immunol Cell Biol 1988;66: 69-77.

34. Rubinstein E, Urso I, Boucheix C, Carroll RC. Platelet activation by cross-linking HLA Class I molecules and Fc receptor. Blood 1992;79:2901-8.

35. Baum C, Dullmann J, Li Z, Fehse B, Meyer J, Williams DA, von Kalle C. Side effects of retroviral gene transfer into hematopoietic stem cells. Blood 2003;101:2099-114.

36. Cavazzana-Calvo M, Fischer A. Gene therapy for severe combined immunodeficiency: are we there yet? J Clin Invest 2007;117:1456-65.

37. Ott MG, Schmidt M, Schwarzwaelder K, Stein S, Siler U, Koehl U, Glimm H, Kühlcke K, Schilz A, Kunkel H, Naundorf S, Brinkmann A, Deichmann A, Fischer M, Ball C, Pilz I, Dunbar C, Du Y, Jenkins NA, Copeland NG, Lüthi U, Hassan M, Thrasher AJ, Hoelzer D, von Kalle C, Seger R, Grez M. Correction of X-linked chronic granulomatous disease by gene therapy, augmented by insertional activation of MDS1-EVI1, PRDM16 or SETBP1. Nat Med 2006;12:401-9. 